\title{
Los Sistemas Productivos Regionales desde la perspectiva del Análisis de Redes
}

\author{
María Semitiel García y Pedro Noguera Méndez. Departamento de Economía \\ Aplicada. Universidad de Murcia (España) ${ }^{1}$
}

\section{Resumen}

En este trabajo se presenta una metodología de análisis de las relaciones industriales y de los sistemas productivos basada en el análisis de redes sociales, y en los conceptos de embeddedness (Granovetter, 1973), sistema productivo (Wilkinson, 1985) y capital social. Tras el examen y discusión de esta perspectiva metodológica, se realiza una aplicación al estudio de las relaciones industriales en el País Vasco (España). La aplicación del análisis de redes sociales en la investigación económica se encuentra todavía en una fase mucho menos avanzada que en otros ámbitos de las ciencias sociales, como la psicología, la antropología o la sociología. Esto obedece, principalmente, a que el paradigma neoclásico, que es la corriente dominante de la economía, no considera relevantes ni el carácter social ni el carácter relacional de los agentes, a pesar de que todas las corrientes de la economía asumen su pertenencia al campo de las ciencias sociales. En este trabajo las relaciones constituyen el centro del análisis y son consideradas en un sistema productivo concreto, delimitado desde la perspectiva geográfica, económica e histórica. El concepto de sistema productivo contempla no sólo las relaciones mercantiles que tienen lugar entre las empresas, que son utilizadas para identificar los sistemas de producción, sino también las no mercantiles. Estas relaciones también se establecen con otros tipos de instituciones tales como parques tecnológicos, institutos de investigación o universidades. También las relaciones informales entre los actores deben ser incluidas, junto a las relaciones formales, para analizar un sistema productivo completo. El análisis de la estructura relacional se realiza considerando su contexto, es decir, contemplando sus características geográficas, históricas y sociales, que ayudan a comprender mejor sus características.

Palabras clave: sistemas productivos regionales, análisis de redes, capital social, relaciones industriales.

\footnotetext{
Abstract

This paper discuss a methodology to analyse industrial relationships based on social network analysis and on the concepts of embeddedness (Granovetter, 1973), productive system (Wilkinson, 1985) and social capital. Once the perspective is

${ }^{1}$ Enviar correspondencia a: Departamento de Economía Aplicada. Universidad de Murcia. Campus de Espinardo 30100, Murcia, España; mariase@um.es; pedrono@um.es
} 
discussed it is applied to study industrial relationships in the Basque Country (Spain).

The application of the social network analysis to economic research is at the moment at its initial stages, although it has been widely applied in sociology and psychology. The dominance of the neoclassical mainstream paradigm in economics, based on principles and developing theories that do not consider as relevant the social and relational character of agents and systems, is among the main reasons. However, all the perspectives, even the neoclassical, assume that economics is a social science.

In this paper relationships are the focus of the analysis and they are considered for a particular productive system, geographically and historically specified. The concept of productive system is understood by considering mercantile and nonmercantile relationships among the firms constituting a particular industrial sector. Moreover, relationships are linking firms, and economic sectors at a more macro level, with other types of institutions such as technology parks, research institutes and universities. At the same time, informal relationships among actors should also be included, together with the formal links, to analyse a complete productive system. The whole relational structure is analysed considering its context, and therefore allowing for the consideration of geographical, historical and social peculiarities, that are necessary for a better understanding of its characteristics.

Main topics: Regional productive systems, social network analysis, social capital, industrial relations.

\section{Introducción}

La perspectiva de redes implica la aceptación de un enfoque metodológico basado en el estudio de sistemas socio-económicos, formados por las relaciones mantenidas entre los actores que los componen. En estos sistemas, las relaciones entre los agentes (empresas, instituciones, individuos) constituyen la base de los intercambios, mercantiles o de otro tipo (información, conocimiento, etc.). Por este motivo, el estudio de sus estructuras es crucial para comprender el funcionamiento de las economías y para diseñar estrategias de crecimiento y desarrollo.

Además, la dimensión territorial es de gran importancia, puesto que la estructura relacional que conforma los sistemas socio-económicos se localiza en espacios geográficos concretos, en los que fluyen conocimiento, información e innovación y en los que se desarrollan relaciones basadas en la confianza. 
Desde esta perspectiva, se presenta una metodología de estudio de sistemas de producción y sistemas productivos regionales, así como una aplicación. Los primeros están formados por las relaciones técnicas mantenidas por las ramas productivas, y por tanto por las empresas, que intercambian bienes y servicios para completar sus procesos productivos. Los segundos se componen por los sistemas de producción y por las relaciones formales e informales mantenidas entre las empresas y el resto de instituciones regionales.

Los sistemas de producción y productivos se estudian en este trabajo en el caso concreto del País Vasco. La estructura de estos sistemas se analiza mediante el uso de las tablas input-output regionales, de información cuantitativa y cualitativa adicional y de los instrumentos desarrollados por el análisis de redes sociales.

\section{La perspectiva de redes}

La perspectiva neoclásica es, desde hace décadas, la corriente dominante de la economía teórica, que es la que, principal o exclusivamente, se imparte en las Universidades de todo el Mundo. Esta teoría está basada en un conjunto de supuestos restrictivos con el fin de alcanzar cierta abstracción, lo que al mismo tiempo ha facilitado el desarrollo de modelos matemáticos explicativos del comportamiento de los agentes económicos. Entre esos fundamentos de la teoría se encuentra el supuesto de que los agentes económicos son individuos racionales que intentan maximizar su bienestar. La utilidad y el beneficio constituyen las expresiones del bienestar de consumidores y productores, respectivamente. Además, consumidores y productores adoptan sus decisiones de forma individual e independiente de las decisiones adoptadas por los restantes individuos, como si fueran 'islas humanas'?

\footnotetext{
${ }^{2}$ En la corriente principal de la economía existen algunas aportaciones que son excepciones, que han considerado la existencia de relaciones entre los agentes. Es el caso de los modelos que expresan las reacciones de las empresas (Cournot, Bertrand) y la teoría de juegos (Nash).
} 
La elusión de las relaciones sociales supone ignorar el carácter social de los agentes económicos, es decir, del comportamiento humano, que constituye la base de los sistemas económicos al nivel más micro. Sin embargo, la realidad nos enseña que todos los agentes económicos son actores sociales que adoptan sus decisiones inmersos en una red de relaciones sociales que proporciona oportunidades, pero también restricciones, dependiendo de cuál sea la posición que ocupen en ella. En este sentido, conviene destacar la conexión establecida por Granovetter (1973) entre la economía y la sociología con el concepto embeddedness, así como por Coleman (1988) a partir del concepto de Capital Social, que será examinado más adelante.

La perspectiva de redes constituye una metodología de investigación donde los agentes son estudiados a partir de las relaciones que mantienen, para lo que ha desarrollado conceptos y herramientas analíticas apropiadas ${ }^{3}$. Su carácter distintivo radica en su perspectiva estructuralista, y en el hecho de que sitúa a las relaciones, y a las estructuras que éstas forman, en el foco de su atención; en contraposición al análisis habitual centrado en el examen de los atributos o características de las unidades de estudio. Wasserman y Faust (1999) han señalado que los principios centrales que subyacen a la perspectiva de redes son, primero, que los actores y sus acciones son consideradas interdependientes; segundo, que las ligazones entre los actores sirven para transferir recursos materiales e inmateriales; tercero, los modelos estudian la estructura relacional de los agentes, contemplándola como un marco condicionante, proveedor de oportunidades pero también de restricciones; y cuarto, que los modelos de redes se ocupan principalmente de conceptos sociales, políticos, económicos y estructurales, que definen patrones permanentes de relaciones entre los actores. Los agentes susceptibles de ser estudiados bajo este enfoque son muy diversos: individuos, empresas, instituciones, regiones, organizaciones,

\footnotetext{
${ }^{3}$ Entre esos conceptos pueden señalarse: puentes, relaciones débiles y relaciones fuertes (Granovetter, 1973), agujeros estructurales (Burt, 1992), capital social (Putnam, 1993), embeddedness (Granovetter, 1985), cliques, redes, centralidad, cohesión, densidad, etc. Dos manuales básicos, mucho más completo el segundo que el primero, son: Degenne y Forsé (1999) y Wasserman y Faust (1999).
} 
etc. pudiendo centrarse el estudio en el análisis de las relaciones mantenidas por uno o varios agentes, o en las estructuras relacionales que definen determinados grupos o colectivos.

La perspectiva de redes, que surgió en el ámbito de la antropología y de la psicología social (Moreno, 1934; Barnes, 1954), ha encontrado aplicaciones en campos muy diversos como la sociología, la política, la medicina y, más recientemente y en menor medida, en la economía. Desde nuestro punto de vista, el análisis de redes proporciona una plataforma muy adecuada para el análisis económico, puesto que permite reintroducir 'lo social', es decir, el contexto social en el que los agentes se desenvuelven, obligando al investigador a mantener una perspectiva amplia e interdisciplinar. Resulta cuando menos sorprendente que, aunque la pertenencia de la economía al extenso campo de las ciencias sociales sea aceptada por toda la comunidad científica, la tradición dominante sólo reconozca como un principio general, la importancia de las relaciones sociales y el carácter social de la economía 4 . En efecto, las relaciones sociales raramente son consideradas por la Teoría Económica, donde encontramos que ni los modelos de Consumo ni los de Producción reflejan el carácter social de la disciplina, considerando, por tanto, que los efectos o la capacidad explicativa del contexto y de las relaciones sociales son irrelevantes o que, al menos, no son fundamentales. Esta situación tiene importantes consecuencias metodológicas. Primero, porque al obviarse el contexto social en el que la acción ocurre, los modelos construidos constituyen deficientes y reduccionistas expresiones de la realidad. Segundo, porque no es cierto que se puedan despreciar los efectos del contexto y de las relaciones sociales sobre el consumo o la producción, por ejemplo. Ni las actividades de consumo, ni la innovación, ni las decisiones de producción que adoptan las empresas son actos puramente individuales e independientes de las decisiones que otros agentes toman, ni tampoco es cierto que la maximización de la utilidad o del beneficio constituyan el único objetivo. Es decir, los agentes económicos son actores

\footnotetext{
${ }^{4}$ Bordieu ha destacado que ni el componente social ni el histórico son contemplados por la teoría neoclásica; aunque sus palabras, de difícil traducción, son más apropiadas: "this theory is desocialised and dehistoricised".
} 
sociales que toman sus decisiones condicionados por la 'red' en la que se encuentran inmersos que, al mismo tiempo, es origen de importantes activos. En este contexto se puede entender el concepto de Capital Social, un recurso que emerge de la estructura relacional existente y que, junto al Capital Físico y al Capital Humano, puede promover el desarrollo económico o el progreso e impulsar la eficiencia de las organizaciones empresariales o no empresariales.

Existen muchas definiciones de Capital Social, entre las que se puede destacar la de Robert Putnam, probablemente la más conocida, aunque muy criticada por incluir en ella uno de sus efectos (la confianza) y porque no contempla la existencia de Capital Social negativo. Para Putnam (1993) el Capital Social hace referencia a las características de la organización social, tales como redes, normas y confianza, que facilitan la coordinación y la cooperación para alcanzar un beneficio mutuo. Más recientemente Putnam (2002) ha definido el Capital Social como las redes sociales y las normas de reciprocidad asociadas a ellas. Para Coleman (1990), el Capital Social está constituido por el conjunto de relaciones disponibles en cualquier momento por parte de sujetos individuales o colectivos, siendo por tanto las relaciones sociales la base del Capital Social. El reciente interés por el Capital Social y por el marco institucional se puede relacionar con las importantes dificultades que atraviesan muchos de los países que integraron el bloque de economías socialistas, en su proceso de transición hacia la economía de mercado (Woolcock, 2001; Putnam y Goss, 2002). La trascendencia de este interés queda bien reflejada en la iniciativa lanzada en 1996 por el Banco Mundial o las publicaciones e investigaciones promovidas por la $\mathrm{OCDE}^{5}$.

\section{La dimensión territorial y social de la producción y del progreso técnico}

\footnotetext{
${ }^{5}$ Los objetivos y resultados alcanzados por 'The Initiative on Defining, Monitoring and Measuring Social Capital' (Social Capital Initiative) del Banco Mundial, pueden consultarse en su web, encontrándose numerosos trabajos. Por su parte, la OCDE también ha financiado reuniones internacionales e investigaciones sobre Capital Social, como puede constatarse en el trabajo editado por Helliwell (2001).
} 
Teniendo presente lo anterior se puede comprender la necesidad de incluir la dimensión social en el análisis de todos los procesos y actividades económicas; esto ha sido destacado, entre otros, por Noguera y Semitiel (2003), al examinar cuestiones consideradas fundamentales por la disciplina económica, como la innovación, la consecución de la eficiencia, la producción y el consumo, desde la perspectiva de la estructura relacional y del Capital Social

Por otra parte, la dimensión espacial de los procesos económicos no es circunstancial, ni cabe concebirla como el simple resultado del origen de la información utilizada: nacional, regional o local. El territorio se puede concebir como un espacio que se define y se reproduce como una red, o un conjunto de ellas, constituida por nodos -instituciones, empresas, organizaciones- entre los que se registran flujos que definen determinadas estructuras que evolucionan, donde existen variadas posiciones (dependencia, poder, estratégicas, etc.) ocupadas por instituciones empresariales o no empresariales $y$, desde otra perspectiva, por los diversos espacios contenidos en la totalidad del territorio (centro y periferia, por ejemplo).

Numerosos trabajos han constatado la sensible variabilidad del crecimiento y del desarrollo económico entre las regiones que integran un país. 'Esto sugiere que muchos de los determinantes esenciales de los resultados económicos se encuentran a nivel regional' (Porter, 2003:550). También Scott y Storper (2003:580) han destacado el papel que ciudades y regiones desempeñan en los procesos de desarrollo. Pueden indicarse tres mecanismos a través de los cuales la dimensión territorial se revela crucial: economías de aglomeración, confianza y procesos de innovación.

El primero de ellos es, sin duda, el más conocido de los tres: la proximidad a recursos, a factores productivos o a mercados, así como la concentración de flujos de información, constituyen fuentes de las ventajas competitivas que pueden alcanzar las empresas localizadas en determinadas áreas o ciudades (Marshall, 1920). El contacto directo y la proximidad facilitan el establecimiento de relaciones de confianza, sobre las que pueden 
construirse estrategias de cooperación y coordinación. La coordinación evita el oportunismo, reduciendo, en consecuencia, los costes de transacción (Williamson, 1983). Además, atendiendo a lo señalado por Ouchi (1980) y Bolton et al (1994) determinadas estructuras relacionales basadas en la confianza que facilitan la externalización de tareas, así como otras muy cohesionadas basadas además en la reciprocidad y en creencias y valores comunes, son sistemas organizacionales superiores, donde el oportunismo es improbable y donde la equidad puede ser alcanzada con costes de transacción relativamente bajos.

La proximidad, o el contacto frecuente, es fundamental para que se produzca la transmisión del conocimiento en los procesos de innovación, particularmente cuando se trata de conocimiento tácito (Gorman, 2002; Baptista, 2000; Rallet y Torre, 1999). Resulta revelador confrontar la concepción del progreso técnico de la economía neoclásica con la propuesta, entre otras, por la corriente institucionalista que, desde nuestra perspectiva, es claramente superior. Pues frente a la perspectiva lineal y sin referencias a lo social y al territorio que presentan los modelos neoclásicos, actualmente numerosos investigadores han destacado que los procesos de innovación son básicamente procesos sociales, que se desarrollan en un proceso interactivo e inmersos en un contexto social, cultural, institucional y territorial (Lundvall, 1992; Morgan 1997; Asheim y Dunford, 1997). En este planteamiento, las relaciones sociales, el contexto institucional, así como el espacio geográfico, no son cuestiones secundarias, sino que son elementos fundamentales e imprescindibles para comprender cómo funcionan y cómo se generan los procesos de innovación. Los procesos de crecimiento e industrialización implican la transformación del territorio y la emergencia de nuevos espacios de aprendizaje (Lundvall y Johnson, 1994; Storper, 1995). La existencia de espacios de interacción entre las empresas, y entre éstas y otras instituciones, se ha revelado fundamental para los procesos de innovación $y$, en general, para el progreso económico. Los conceptos 'sistema nacional (o regional) de innovación' y 'economías (o regiones) de aprendizaje' remiten a espacios donde 'el conocimiento es el recurso más estratégico y el aprendizaje el proceso más importante' (Lundvall, 1994 y 
1996). Estos conceptos también remiten a una nueva perspectiva teórica de la economía que, según Morgan (1997), es el resultado de la creciente confluencia entre la geografía económica y los estudios sobre la innovación, principalmente por parte de la economía institucionalista. En consecuencia, y resumiendo lo anterior, la perspectiva metodológica de este trabajo es relacional, estructural y regional.

\section{Sistemas de producción y sistemas productivos regionales}

Existe una amplia literatura económica sobre la identificación y caracterización de grupos de empresas o de ramas productivas relacionadas en la obtención de un bien o servicio determinado. Estos grupos o sistemas son definidos a partir de las relaciones que mantienen, que pueden estar basadas, por ejemplo, en el intercambio de bienes intermedios y de capital: si la empresa A proporciona bienes de inversión a las empresas B y C, y éstas a su vez venden a $D$ determinados inputs que ésta precisa, las empresas $A, B, C$ y $D$ podrían formar un grupo o sistema determinado, tal vez un sistema regional de producción. Existen diversos conceptos para nombrar estos agrupamientos de ramas o de empresas relacionadas, que se diferencian principalmente por la importancia o el papel que otorgan a las relaciones sociales, a la localización y a las instituciones no empresariales: 'sistema productivo' (Wilkinson, 1983; Lawson, 1999), 'sistema de producción' (Storper y Harrison, 1991), 'sistema productivo regional' (Asheim y Dunford, 1997), 'cluster regional de empresas' (Lawson, 1999), 'distrito industrial' y 'cluster industrial' (Gordon y McCann, 2000; Fesser y Bergman, 2000), 'complejo industrial' (Streit, 1969), 'technological regimes' (Breschi, 2000).

En este trabajo se utilizarán dos conceptos que servirán para la identificación de sendos agregados en el ámbito regional: sistema de producción y sistema productivo. Un sistema de producción es un conjunto de unidades de producción ligadas a través de su estructura de relaciones input-output en un ámbito espacial determinado. Como, por ejemplo, el sistema de producción agroalimentario, integrado, entre otras unidades productivas, por las empresas o explotaciones agrarias, las industrias de 
suministros de medios de producción (semillas, fertilizantes, combustibles, etc.), las industrias de conservas y de transformación del output agrario, etc. Es decir, un sistema de producción está determinado, fundamentalmente, por las relaciones técnicas de producción, aunque, a nivel regional, cuando sólo se consideran los flujos input-output interiores, la especialización y el nivel de desarrollo condicionarán, no sólo la naturaleza de los sistemas existentes, sino también su configuración. Asimismo es conveniente destacar que los sistemas de producción pueden ser definidos a partir de las empresas, como mínimas unidades de análisis, pero también a partir de ramas productivas, que agrupan los intercambios realizados por empresas que producen bienes o servicios similares. Por último, una empresa, o una rama productiva, puede formar parte de más de un sistema de producción, existiendo por ello solapamientos entre los sistemas de producción.

De acuerdo con la definición anterior, los sistemas de producción pueden ser identificados a partir del agrupamiento de ramas productivas mutuamente dependientes, por encontrarse implicadas en relaciones de intercambio importantes tanto para la rama oferente como para la demandante. Estas relaciones sirven, asimismo, al proceso de innovación a través de procesos de aprendizaje interactivos y de tecnología incorporada

El concepto sistema productivo es mucho más amplio, puesto que incluye, además de las relaciones comerciales, las relaciones formales e informales entre empresas e instituciones no empresariales. Un sistema productivo es concebido, por tanto, como el conjunto de instituciones empresariales y no empresariales, inmersas en una compleja red de relaciones técnicas, económicas, sociales y políticas, en un período y tiempo determinados.

Para Wilkinson (1983) el concepto 'sistema productivo' es aún más amplio. En realidad, lo que Wilkinson plantea es una severa crítica al análisis 'unidimensional' que se realiza con demasiada frecuencia por parte de la corriente principal de la economía, planteando una alternativa metodológica que, por otra parte, no supone sino la revisión de la base epistemológica de los economistas clásicos. Por lo tanto, en esta metodología han de estar 
necesariamente presentes, en la investigación, las perspectivas histórica, sociológica y política, además de la económica.

La figura 1 facilita la comprensión de algunos de los conceptos utilizados aquí. Los sistemas de producción están integrados por ramas productivas que, a un nivel más microeconómico, se componen de empresas. Una rama productiva puede formar parte de más de un sistema de producción. La determinación de las ramas que integran un sistema de producción se realiza a partir de la intensidad de las relaciones de compra y venta que mantienen entre sí, y será explicada en el apartado siguiente. El concepto 'sistema productivo' es más amplio, incluyendo el anterior. Por ello, un sistema productivo está integrado, además de por las ramas o empresas estrechamente relacionadas, por aquellas instituciones públicas o privadas que participan en alguna de las fases de elaboración del producto o de prestación de servicios, y cuya actividad incide en los resultados de los sectores relacionados. A un nivel más agregado se encuentra la estructura económica regional que, junto a otras, conforman las estructuras económicas nacionales, y éstas a su vez las de la economía mundial. 


\section{Figura 1.- Conceptos y dimensiones básicas del análisis regional}

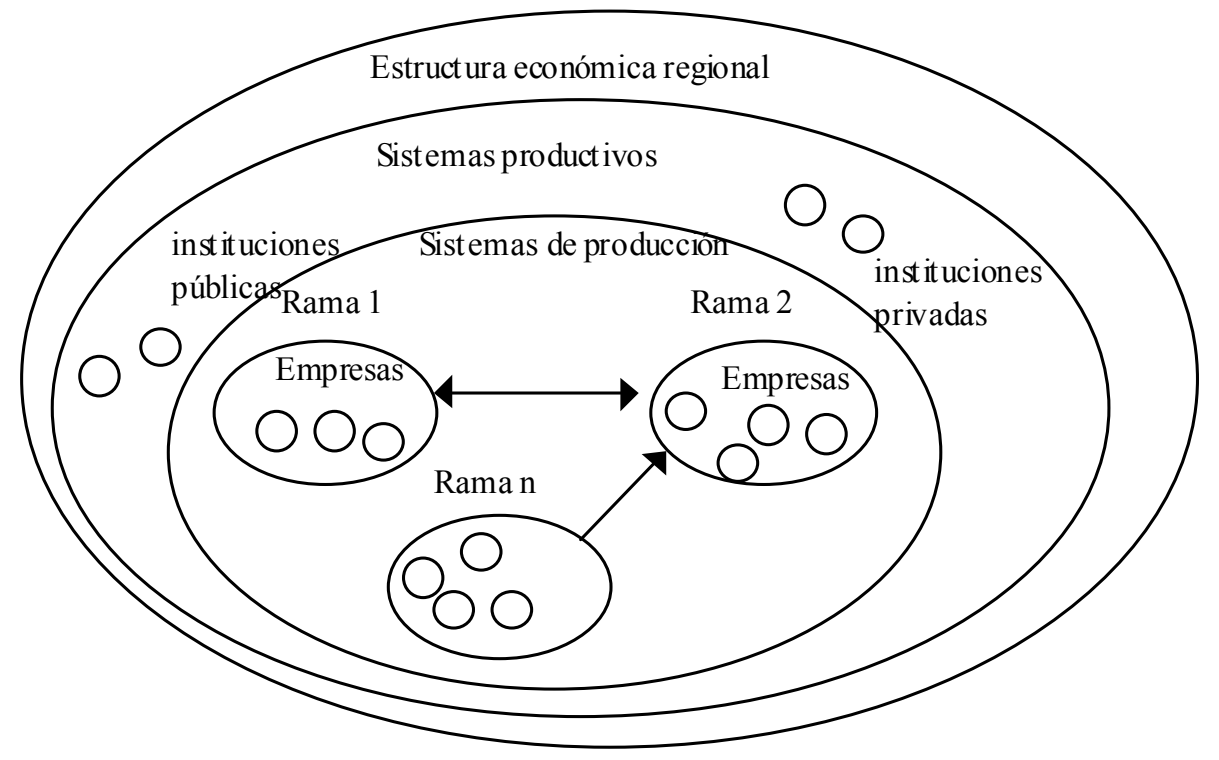

\section{Análisis estructural del sistema económico del País Vasco. Sistemas de producción y sistemas productivos}

En este último apartado se presenta una aplicación de la perspectiva metodológica defendida en las páginas anteriores, al estudio de la estructura económica del País Vasco. Es conveniente destacar el carácter incompleto de esta aplicación puesto que, si bien los principales aspectos del análisis de redes se encuentran presentes, las limitaciones de espacio nos impiden entrar a examinar el contexto histórico, político y económico en el que se ha desarrollado la economía vasca. También estas limitaciones condicionan el nivel de profundización del análisis relacional de la estructura vasca de transacciones intermedias, así como el examen del sistema productivo que, en mayor medida, representa su especialización y su tradición industrial, la que conforman los sectores siderometalúrgico y de fabricación de máquinas-herramientas, es decir, el Sistema Productivo Metal-Mecánico.

Los datos utilizados para realizar el examen de la red regional de relaciones económicas son los flujos regionales input-output, considerando sólo los que se registran inter-ramas y no intra-ramas, y sólo los valores interiores, en vez de los nacionales o los totales. Esto ha sido así atendiendo a que el 
objetivo consiste en estudiar los sistemas productivos regionales, y la utilización de otro tipo de información que no fuera exclusivamente la interior, daría lugar a la consideración de relaciones inter-ramas no relevantes desde el punto de vista interior, así como la determinación de sistemas productivos que no tendrían un carácter regional.

Es importante comprender que la naturaleza de la información utilizada tiene importantes ventajas, pero también algunos inconvenientes desde la perspectiva de los objetivos que se pretenden alcanzar. Concretamente, la imposibilidad de considerar los flujos intersectoriales de bienes de capital, junto a las transacciones intermedias, constituye, desde nuestro punto de vista, la principal deficiencia de la información que proporcionan las Tablas Input-Output. Esto significa, por ejemplo, que las relaciones mantenidas por las ramas que producen maquinaria y bienes de equipo con otras ramas, no estarán suficientemente representadas. Es decir, puede suceder que ramas situadas en posiciones centrales en un determinado sistema económico debido a su función suministradora de bienes de capital, ocupen posiciones periféricas al sistema cuando sólo las transacciones intermedias son consideradas.

\subsection{Identificación de sistemas regionales de producción}

La identificación de las relaciones significativas $y$, a partir de ellas, de los sistemas de producción, se ha realizado determinando, primero, las relaciones input-output importantes desde la perspectiva de las ramas implicadas. De este modo, las ligazones seleccionadas y, por tanto, los nodos o ramas correspondientes, representan relaciones mutuamente dependientes. Este proceso se ha llevado a cabo aplicando un algoritmo para la identificación de clusters, basado en Peeters et al (2001) que busca las relaciones 'hacia adelante' y 'hacia atrás' más significativas de cada rama, a partir de los coeficientes de compras y de ventas relativos.

Más concretamente, los algoritmos se han aplicado sobre las matrices de coeficientes relativos de compras y de ventas intermedias interiores (con respecto a las compras o a las ventas intermedias totales de cada rama), 
calculados sobre la tabla input output del País Vasco en 1995, que previamente ha sido transformada para posibilitar su comparación con la de 1980. En el análisis de las relaciones 'hacia adelante' primero se han seleccionado las relaciones más importantes de cada rama vendedora, aplicando un filtro exigente: el 10\%. Es decir, en esta etapa, sólo se consideran aquellas relaciones que representan al menos el $10 \%$ de las ventas intermedias de la rama vendedora. Pero no todas estas relaciones van a ser seleccionadas puesto que para ello se requiere que, simultáneamente, estas relaciones intermedias alcancen, como mínimo el $5 \%$ de las compras intermedias totales de las ramas compradoras implicadas en las transacciones. En otros términos, la selección de las relaciones 'hacia adelante', es muy exigente con respecto a la rama vendedora (el filtro elimina las transacciones que no alcanzan como mínimo el $10 \%$ de sus ventas) pero no lo es tanto desde la perspectiva de la rama compradora (filtro del 5\%). En cualquier caso, el algoritmo exige la existencia de una dependencia mutua, mucho menor para la rama compradora.

El análisis de las relaciones 'hacia atrás' se inicia con la selección de las relaciones más importantes desde la perspectiva de las compras, aplicando un filtro del $10 \%$. Esto significa que la ligazón entre dos ramas será considerada siempre y cuando dicha transacción intermedia represente al menos el $10 \%$ de las compras intermedias realizadas por la rama compradora. A continuación se aplica un filtro del $5 \%$ sobre estas relaciones con el fin de eliminar aquellas que no alcancen dicho porcentaje desde la perspectiva de las ventas. El algoritmo, en realidad, se ha aplicado para distintos filtros, y se han considerado también criterios de máximos. En este trabajo se han incluido tanto las relaciones 'hacia atrás' como las existentes 'hacia adelante'. El conjunto de las relaciones intermedias seleccionadas se ha representado en la figura 2, constituyendo la base sobre la que, utilizando además información cualitativa, se han delimitado los sistemas de producción (figura 3 ). 


\subsection{Análisis de la estructura de la red de transacciones intermedias}

Existen numerosas medidas y conceptos que permiten estudiar y caracterizar, desde un enfoque relacional, tanto los nodos como el conjunto de la red. A continuación se presenta un breve análisis en el que se ha hecho uso de dos conceptos básicos del análisis de redes: centralidad (grado) y estructura centro-periferia. El valor del grado se calcula a partir del número de relaciones que cada rama (nodo) mantiene con las restantes. Se distingue entre indegree y outdegree. El primero se corresponde con el número de relaciones que implican un cierto flujo de 'entrada', mientras que el outdegree hace referencia al número de relaciones que implican un flujo de salida. Es decir, en nuestro caso (cuadro 1) si la rama 35 tiene un outdegree igual a 5 en 1995, esto significa que esta rama vende a cinco ramas. Más concretamente significa, teniendo en cuenta los filtros que se han aplicado, que la rama 35 mantiene ligazones significativas de ventas con cinco ramas. En el cuadro 1 se presentan estas medidas del grado de todas las ramas en 1980 y 1995, así como las variaciones experimentadas en el transcurso del período contemplado. El criterio de ordenación de las ramas que se ha utilizado ha sido el grado total en 1995.

La destacada presencia y avance de las actividades terciarias en las economías desarrolladas también se constata desde una perspectiva relacional. En efecto, entre los cuatro primeros lugares se encuentran tres importantes ramas de servicios que además incrementan sus respectivos grados entre 1980 y 1995: 35'Reparación y comercio', 44'Servicios a empresas' y 36'Hostelería'. También la especialización en el trabajo de los metales y la fabricación de maquinaria es patente.

El procedimiento para determinar las ramas que forman el núcleo en una posible estructura centro-periferia se basa en un algoritmo que ajusta la matriz de datos a una matriz 'modelo' o ideal que representaría una estructura centro-periferia perfecta, en la que los nodos (ramas) del núcleo mantienen todos ellos relaciones entre sí (Borgatti y Everett, 1999). Los resultados obtenidos se ofrecen en el cuadro 2 . 
Desde la perpectiva que nos ocupa, las ramas que integran el núcleo o centro del sistema económico regional, de mayor a menor puntuación de coreness $^{6}$ alcanzada en la aplicación del correspondiente algoritmo, son: 35'Reparación y comercio', 44'Servicios a empresas', 15'Productos metálicos', 40'Actividades anexas al transporte', 34'Construcción', 36'Hostelería', 9'Siderurgia y fundición', 41'Correos y telecomunicaciones', 37'Otro transporte terrestre' y 17'Máquinas de oficina'. En la figura 2, el círculo que se ha dibujado incluye estas diez ramas centrales de la economía regional.

Como puede comprobarse, entre las ramas indicadas no se encuentran algunas de las que presentan mayor número de relaciones, como es el caso de las ramas 1'Agricultura' y 2'Ganadería'. En este caso, lo que se observa es la clara delimitación del Sistema Agrolimentario, que aparece relativamente aislado del resto de la estructura económica regional, unido tan sólo al sistema económico regional a través de dos ramas: 36'Hostelería' y 30'Industria del papel'. Por ello, tanto la rama 'agricultura' como la rama 'ganadería', aunque mantienen un elevado número de relaciones de compra y de venta con otras ramas, al encontrarse éstas a su vez tan aisladas, no forman parte del Centro del Sistema. Son ramas centrales pero de su propio sistema: el sistema agrolimentario. De hecho, la puntuación coreness que alcanzan estas ramas (también la pesca) es cero, indicando claramente la posición periférica que ocupan.

En el otro extremo se encuentran las ramas que integran la periferia, que serían, entre otras, las catorce últimas del cuadro 1. Aunque esto no debe hacernos pensar que este cuadro nos puede servir para determinar las posiciones de las ramas en la red. Aunque, lógicamente, las ramas más externas a la economía son aquellas que no mantienen ninguna ligazón significativa con ninguna otra rama regional. Al nivel de los filtros utilizados,

\footnotetext{
${ }^{6}$ Coreness es un término que podría traducirse por 'centralidad' pero esto daría lugar a una confusión con las medidas de centralidad, como las ofrecidas en el cuadro 1. Coreness es una medida que indica, a través de una puntuación, el grado de pertenencia al núcleo (en una estructura centro-periferia), permitiendo establecer un orden jerárquico. Es decir, se trata de una medida de la posición de cada nodo en la red.
} 
esto sucede en 1995 en cinco ocasiones: 8'Minerales metálicos', 12'Vidrio', 27'Tabaco', 33'Otras manufacturas' y 49'Servicio doméstico'. Tras comprobar las puntuaciones coreness, también pueden considerarse ramas periféricas las que como máximo mantienen una relación (grado=1), sin hacer consideraciones sobre si es de entrada o de salida. Nueve ramas se encuentran en esta situación, que son las comprendidas (véase cuadro 1) entre la rama 4'Carbones' y la 47'Educación'. Pero hay más: en realidad la periferia parece extenderse a un número de ramas muy superior, la mayor parte de ellas con puntuaciones nulas o próximas a cero. Dado el elevado número de ramas situadas fuera del núcleo, la variabilidad de sus puntuaciones y con el fin de disponer de una clasificación más precisa, puede ser conveniente distinguir un tercer espacio: la semiperiferia, que ocupa el espacio de transición entre la periferia y el centro del sistema económico regional (cuadro 2).

\subsection{El sistema productivo Metal-Mecánico Vasco}

Las ramas que componen el sistema de producción metal-mecánico (figura 3) son: 35'Reparación y Comercio', 44'Servicios a empresas', 9'Siderurgia y fundiciones', 15'Productos metálicos', 5'Coquerías, petróleo y gas natural', 17'Máquinas de oficina', 6'Electricidad', 10'Metales no férreos', 18 'Vehículos', 7'Gas y agua' y 16'Máquinas para trabajar los metales'.

La larga e intensa especialización regional en los sectores siderometalúrgico y de fabricación de máquinas-herramientas, que constituyen las actividades básicas del sistema de producción metal-mecánico, explica que éste ocupe una posición relativamente central en el sistema económico regional. Tal y como se puede comprobar en las figuras 1 y 2 , cinco de las once ramas que integran su sistema de producción pertenecen al 'Centro' de la estructura económica regional: 'Siderurgia y fundición', 'Productos metálicos', 'Reparaciones y comercio', 'Máquinas de oficina' y 'Servicios a empresas'.

Además, el número de relaciones significativas que mantiene la mayoría de las ramas es muy elevado, alcanzando seis ramas un grado total igual o superior a cinco, y situándose sólo dos en la periferia del sistema económico 
regional; concretamente 'Gas y agua' y la rama 16'Máquinas metales' cuya situación no se corresponde ni con su función provisora de bienes a otras ramas del sector, ni con su importancia.

En efecto, la rama número 16, 'Maquinaria para trabajar los metales', constituye una rama clave en el País Vasco; no sólo atendiendo a su contribución a la producción y al empleo, sino también al estratégico papel que desempeña tal output desde la perspectiva de la especialización productiva regional. Sin embargo, la información que proporciona el cuadro 1 no parece indicar nada de esto. La causa es que las relaciones contempladas por las Tablas Input-Output son intermedias y no finales, y el output de esta rama constituye bien de inversión o de capital. Por ello, su importancia, así como las destacadas ligazones que mantiene respecto a ramas tan significativas como 9'Siderurgia y fundición', 15'Productos metálicos', 34'Construcción' y 17'Máquinas de oficina', entre otras, no se puede comprobar aquí.

La figura 4 constituye una representación esquemática del Sistema Productivo Metal-mecánico del País Vasco. Ésta se deduce una vez que se añaden las relaciones estables que mantienen las empresas pertenecientes a las ramas que componen el sistema de producción y las instituciones públicas y privadas regionales, dedicadas principalmente a la difusión de innovación y tecnología, y a actividades de formación. A partir del estudio cualitativo realizado, se ha podido constatar la percepción que las instituciones empresariales y no empresariales tienen sobre los efectos positivos del establecimiento de relaciones formales e informales. El flujo de información y de conocimiento que se sustenta en relaciones duraderas parece haber incidido en los procesos de innovación y en el nivel de eficiencia del tejido productivo vasco.

Aunque en la figura 4 no se hayan representado las relaciones entre los distintos grupos que forman el sistema, éstas se encuentran presentes prácticamente entre todos ellos. El componente más importante de este sistema, al margen del sistema de producción, es la Red Vasca de Innovación y Tecnología (SARETEK), que incluye a la mayor parte de las 
instituciones con un papel relevante en la difusión y creación de innovación regional.

\section{Conclusiones}

La perspectiva de redes ofrece un enfoque metodológico coherente con el estudio de sistemas socio-económicos regionales basados en la estructura de las relaciones mantenidas entre los actores que los componen. Por este motivo ésta es la perspectiva adoptada en el presente trabajo, en el que se identifican y analizan sistemas de producción y productivos regionales, en el caso concreto del País Vasco.

La red de relaciones comerciales y los sistemas de producción han sido identificados a partir de la información ofrecida por las tablas input-output regionales, a las que se ha aplicado un algoritmo que permite determinar las relaciones más importantes, y por tanto las ramas productivas más arraigadas en el sistema económico vasco de acuerdo a las transacciones intermedias llevadas a cabo. El análisis se ha realizado para los años 1980 y 1995 y ha permitido identificar un grupo de ramas integrantes del sistema metal-mecánico que ocupan una posición claramente central en la estructura productiva regional.

Otra información cuantitativa y cualitativa se ha añadido para formar el sistema productivo metal-mecánico, que incluye un conjunto de instituciones públicas y privadas que mantienen relaciones formales $e$ informales entre sí y con las empresas, lo cual favorece el flujo de información, conocimiento e innovaciones, con un claro efecto positivo en el crecimiento y el desarrollo regional. 


\section{Anexo: cuadros y figuras.}

Cuadro 1.- La centralidad de las ramas productivas en la estructura económica del País Vasco en 1980 y 1995. Filtro 10/5.

\begin{tabular}{|c|c|c|c|c|c|c|c|c|c|}
\hline & \multicolumn{3}{|c|}{1980} & \multicolumn{3}{|c|}{1995} & \multicolumn{3}{|c|}{ Variación 95/80 } \\
\hline & OutD & $\ln D$ & total & OutD & $\ln D$ & total & Out & In & Total \\
\hline 35"Repar.y comercio" & 3 & 5 & 8 & 5 & 5 & 10 & 2 & 0 & 2 \\
\hline 44"Serv.empresas" & 5 & 3 & 8 & 7 & 2 & 9 & 2 & -1 & 1 \\
\hline 9"Siderurgia,fund" & 4 & 4 & 8 & 2 & 6 & 8 & -2 & 2 & 0 \\
\hline 36"Hostelería" & 1 & 4 & 5 & 2 & 6 & 8 & 1 & 2 & 3 \\
\hline 15"Ptos metálicos" & 5 & 5 & 10 & 4 & 3 & 7 & -1 & -2 & -3 \\
\hline 2"Ganadería" & 4 & 2 & 6 & 5 & 2 & 7 & 1 & 0 & 1 \\
\hline 34"Construcción" & 3 & 5 & 8 & 1 & 5 & 6 & -2 & 0 & -2 \\
\hline 5"Coquerías P\&GN" & 6 & 0 & 6 & 5 & 1 & 6 & -1 & 1 & 0 \\
\hline 1"Agricultura" & 4 & 1 & 5 & 4 & 1 & 5 & 0 & 0 & 0 \\
\hline 17"Máquinas oficina" & 2 & 2 & 4 & 2 & 3 & 5 & 0 & 1 & 1 \\
\hline 39"Tte.mar.,aéreo" & 0 & 3 & 3 & 1 & 4 & 5 & 1 & 1 & 2 \\
\hline 37"Otro tte.ttre" & 2 & 3 & 5 & 1 & 3 & 4 & -1 & 0 & -1 \\
\hline 40"Act.anexas tte" & 3 & 1 & 4 & 3 & 1 & 4 & 0 & 0 & 0 \\
\hline 30"Ind.papel" & 2 & 3 & 5 & 1 & 2 & 3 & -1 & -1 & -2 \\
\hline 6"Electricidad" & 3 & 1 & 4 & 3 & 0 & 3 & 0 & -1 & -1 \\
\hline 26"Bebidas" & 1 & 3 & 4 & 1 & 2 & 3 & 0 & -1 & -1 \\
\hline 10"Metales NF" & 3 & 0 & 3 & 3 & 0 & 3 & 0 & 0 & 0 \\
\hline 13"Ptos.construcción" & 1 & 2 & 3 & 1 & 2 & 3 & 0 & 0 & 0 \\
\hline 25"Otra ind.alim" & 2 & 1 & 3 & 3 & 0 & 3 & 1 & -1 & 0 \\
\hline 41 "Correos y telec" & 2 & 1 & 3 & 3 & 0 & 3 & 1 & -1 & 0 \\
\hline 20"Otro mat.tte" & 1 & 1 & 2 & 2 & 1 & 3 & 1 & 0 & 1 \\
\hline 19"Constr.naval" & 2 & 2 & 4 & 2 & 0 & 2 & 0 & -2 & -2 \\
\hline 42"Interm.finan" & 0 & 4 & 4 & 0 & 2 & 2 & 0 & -2 & -2 \\
\hline 48"Admón.pública" & 1 & 3 & 4 & 0 & 2 & 2 & -1 & -1 & -2 \\
\hline 3"Pesca" & 1 & 2 & 3 & 1 & 1 & 2 & 0 & -1 & -1 \\
\hline 18"Vehículos" & 1 & 1 & 2 & 0 & 2 & 2 & -1 & 1 & 0 \\
\hline 24"Mol,panadería" & 1 & 1 & 2 & 1 & 1 & 2 & 0 & 0 & 0 \\
\hline 32"Ptos.plástico" & 0 & 2 & 2 & 1 & 1 & 2 & 1 & -1 & 0 \\
\hline 43"Seguros" & 1 & 1 & 2 & 0 & 2 & 2 & -1 & 1 & 0 \\
\hline 46"Serv.pers" & 2 & 0 & 2 & 2 & 0 & 2 & 0 & 0 & 0 \\
\hline 21"Ind.cárnica" & 0 & 1 & 1 & 1 & 1 & 2 & 1 & 0 & 1 \\
\hline 45"San.y veterin" & 0 & $\underline{0}$ & 0 & 1 & 1 & 2 & 1 & 1. & 2 \\
\hline 14"Química ag\&ind & 1 & $\overline{2}$ & 3 & 0 & $\overline{1}$ & 1 & -1 & -1 & $-\overline{2}$ \\
\hline 31"Ptos.caucho " & 1 & 1 & 2 & 0 & 1 & 1 & -1 & 0 & -1 \\
\hline 38"Tte.ferrocarril" & 1 & 1 & 2 & 0 & 1 & 1 & -1 & 0 & -1 \\
\hline 4"Carbones" & 1 & 0 & 1 & 1 & 0 & 1 & 0 & 0 & 0 \\
\hline 7"Gas y agua" & 1 & 0 & 1 & 1 & 0 & 1 & 0 & 0 & 0 \\
\hline 11"Cemento, cal" & 1 & 0 & 1 & 1 & 0 & 1 & 0 & 0 & 0 \\
\hline 16"Máquinas metales" & 0 & 1 & 1 & 0 & 1 & 1 & 0 & 0 & 0 \\
\hline 22"Ind.láctea" & 0 & 1 & 1 & 0 & 1 & 1 & 0 & 0 & 0 \\
\hline 23"Cons.pescado" & 0 & 1 & 1 & 0 & 1 & 1 & 0 & 0 & 0 \\
\hline 29"Madera y corcho" & 1 & 0 & 1 & 0 & 1 & 1 & -1 & 1 & 0 \\
\hline 28"Textil y calzado" & 0 & 0 & 0 & 0 & 1 & 1 & 0 & 1 & 1 \\
\hline 47"Educación" & 0 & 0 & 0 & 0 & 1 & 1 & 0 & 1 & 1 \\
\hline 12"Vidrio" & 1 & 0 & 1 & 0 & 0 & 0 & -1 & 0 & -1 \\
\hline 8"Minerales metál" & 0 & 0 & 0 & 0 & 0 & 0 & 0 & 0 & 0 \\
\hline 27"Tabaco" & 0 & 0 & 0 & 0 & 0 & 0 & 0 & 0 & 0 \\
\hline 33"Otras manuf" & 0 & 0 & 0 & 0 & 0 & 0 & 0 & 0 & 0 \\
\hline 49"Serv.doméstico" & 0 & 0 & 0 & 0 & 0 & 0 & 0 & 0 & 0 \\
\hline total & 74 & 74 & 148 & 71 & 71 & 142 & -3 & -3 & -6 \\
\hline
\end{tabular}

Elaboración propia a partir de Eustat:Tablas input ouput 1980 y 1995 del País Vasco. 
Cuadro 2.- Posiciones, en una estructura Centro-Periferia, de las ramas del País Vasco, atendiendo a sus transacciones intermedias interiores en 1995.

\begin{tabular}{cr}
\hline Ramas centrales & Coreness \\
\hline 35 "Repar.y comercio" & 0.581 \\
44 "Serv.empresas" & 0.402 \\
15 "Ptos metálicos" & 0.308 \\
40 "Act.anexas tte" & 0.274 \\
34 "Construcción" & 0.248 \\
36 "Hostelería" & 0.248 \\
9 "Siderurgia,fund" & 0.239 \\
41 "Correos y telec" & 0.215 \\
37 "Otro tte.ttre" & 0.215 \\
17 "Máquinas oficina" & 0.156 \\
\hline
\end{tabular}

\begin{tabular}{lr}
\hline \multicolumn{1}{c}{ Periferia } & coreness \\
\hline 38 "Tte.ferrocarril" & $2.88 \mathrm{E}-10$ \\
33 "Otras manuf" & $6.29 \mathrm{E}-14$ \\
32 "Ptos.plástico" & $1.73 \mathrm{E}-14$ \\
20 "Otro mat.tte" & $2.25 \mathrm{E}-15$ \\
31 "Ptos.caucho " & $5.58 \mathrm{E}-21$ \\
29 "Madera y corcho" & $2.87 \mathrm{E}-24$ \\
28 "Textil y calzado" & $1.90 \mathrm{E}-30$ \\
27 "Tabaco" & $2.26 \mathrm{E}-39$ \\
26 "Bebidas" & $2.80 \mathrm{E}-45$ \\
1 "Agricultura" & 0 \\
2 "Ganadería" & 0 \\
3 "Pesca" & 0 \\
4 "Carbones" & 0 \\
6 "Electricidad" & 0 \\
7 "Gas y agua" & 0 \\
8 "Minerales metál" & 0 \\
11 "Cemento, cal" & 0 \\
12 "Vidrio" & 0 \\
13 "Ptos.construcción" & 0 \\
14 "Química ag\&ind" & 0 \\
16 "Máquinas metales" & 0 \\
18 "Vehículos" & 0 \\
19 "Constr.naval" & 0 \\
21 "Ind.cárnica" & 0 \\
22 "Ind.láctea" & 0 \\
23 "Cons.pescado" & 0 \\
24 "Mol,panadería" & 0 \\
25 "Otra ind.alim" & 0 \\
47 "Educación" & 0 \\
48 "Admón.pública" & 0 \\
49 "Serv.doméstico" & 0 \\
\hline ( Tablas Input Output & 0 \\
\hline
\end{tabular}

Fuente: elaboración propia a partir de Eustat: Tablas Input Output 1980 y 1995 del País Vasco. 
Figura 2. Estructura de relaciones input-output en la economía del País Vasco en 1995.

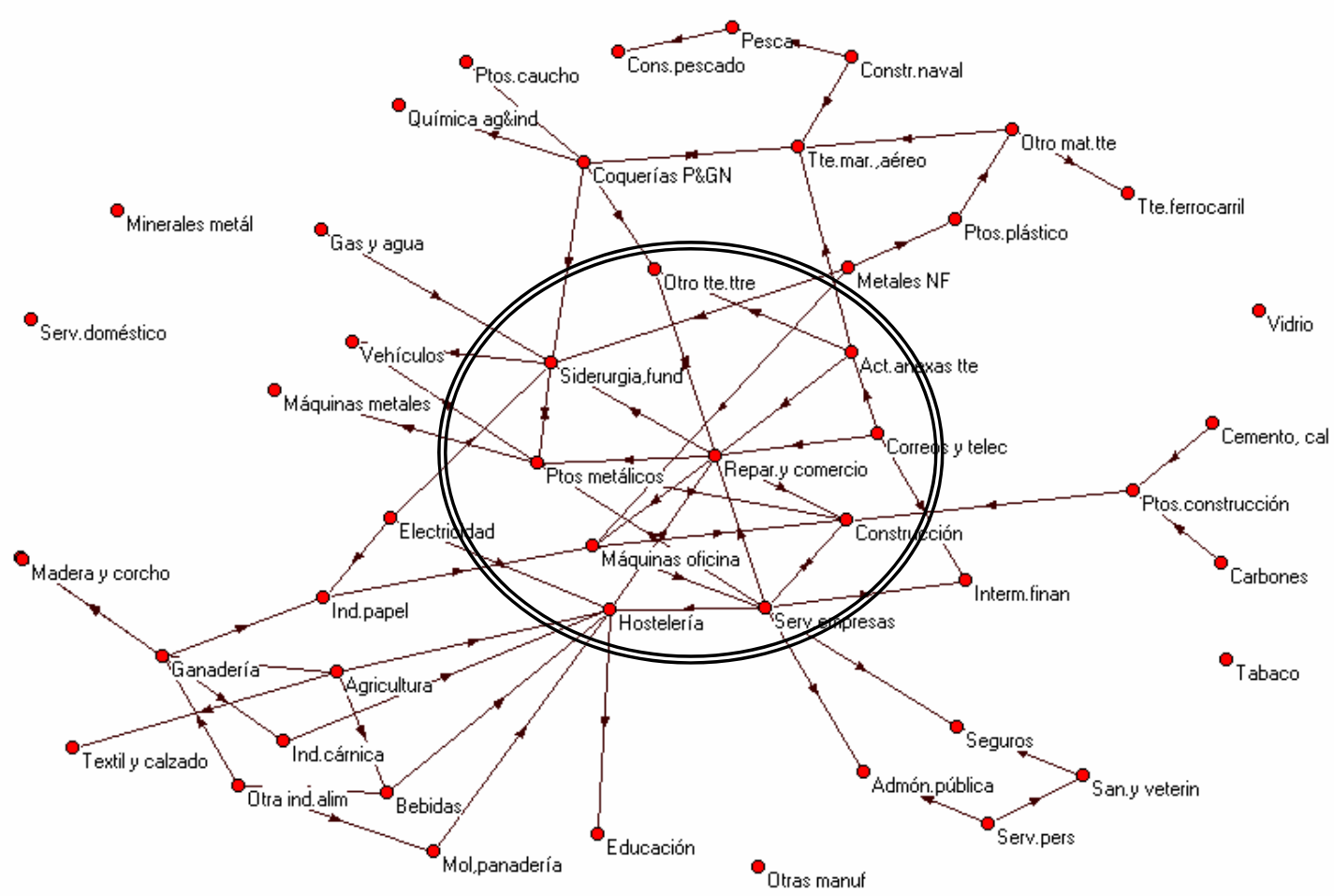

Figura 3. Sistemas de producción del País Vasco. 1995.

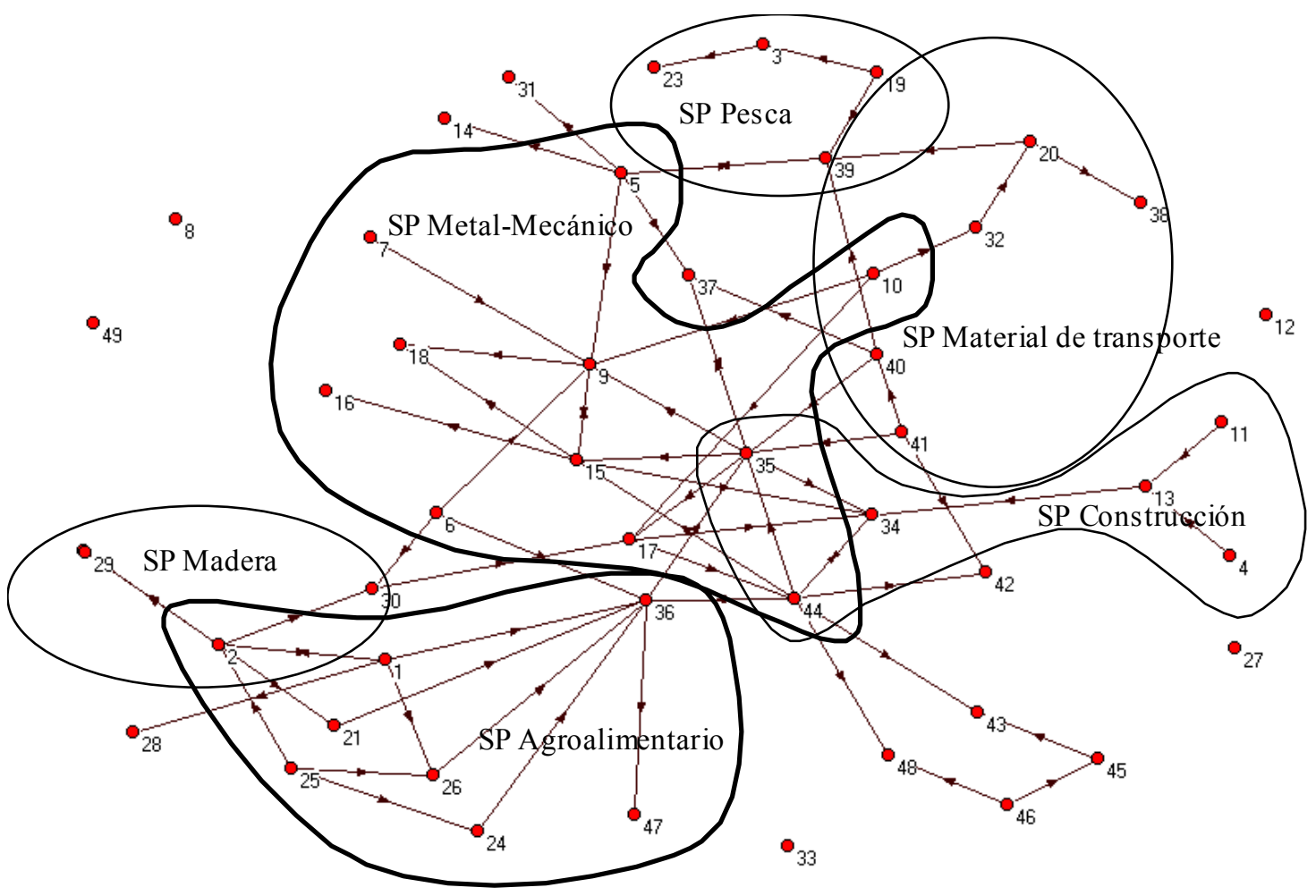


Figura 4.- El Sistema productivo Metal-Mecánico del País Vasco.

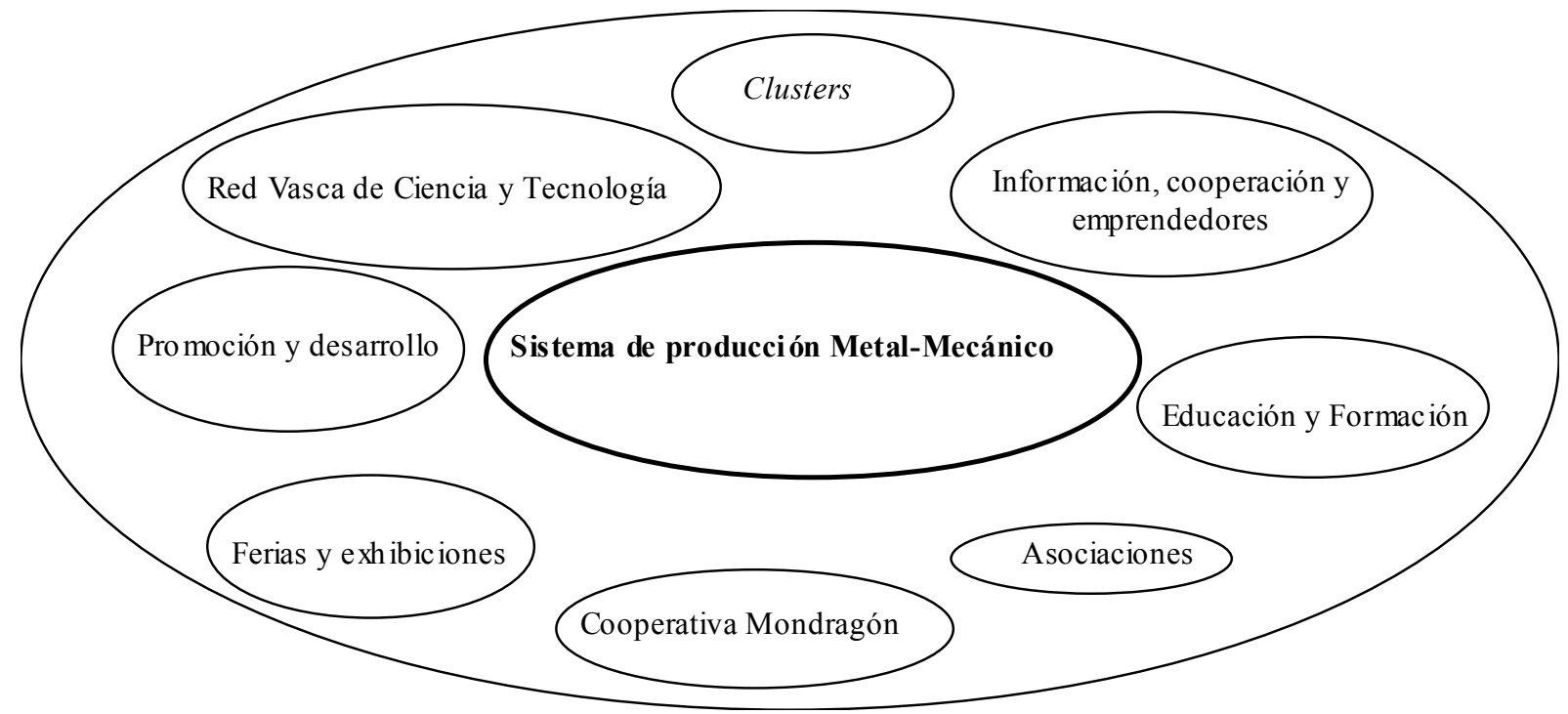

\section{Bibliografía}

Asheim, B. and Dunford, M. (1997): "Regional Futures", Regional Studies, 31,5 , pp. 445-455.

Baptista, R. (2000): "Do Innovations diffuse faster within geographical clusters?", International Journal of Industrial Organization, 18, pp. 518-35.

Barnes, J.A. (1954): "Class and Commitees in a Norwegian island parish". Human Relations 7, pp. 39-58.

Bolton, M. K., Malmrose, R. y Ouchi, W. G. (1994), "The organization of innovation in the United States and Japan: neoclasscial and relational contracting", Journal of Management Studies 31:5, September 1994, pp. 653-79.

Bourdieu, P. (1998): "Utopia of endless exploitation. The essence of neoliberalisme". Le Monde Diplomatique, December.

Borgatti, S.P. y Everett, M.G. (1999): "Models of core-periphery structures". Social Networks 21, pp. 375-395. 
Breschi, S. (2000): "The geography of innovation: a cross-sector analysis", Regional Studies, vol. 34.3, pp. 213-29.

Burt, R. S (1992), Structural holes: the social structure of competition Cambridge, Mass.: Harvard University Press.

Coleman, J. S. (1988): "Social capital in the creation of human capital", American Journal of Sociology, volume 94, issue supplement: organizations and institutions: sociological and economic approaches to the analysis of social structure, S95-S120.

Coleman, J. (1990): Foundations of Social Theory. Cambridge, Harvard University Press.

Degenne, A. and M. Forsé (1999): Introducing Social Networks, Sage Publications.

Feser, E.J. and Bergman, E.M. (2000): "National industry cluster templates: a framework for applied regional cluster analysis", Regional Studies, 34, 1, pp. 1-19.

Gordon, I. R. y McCann, P. (2000): "Industrial clusters: complexes, agglomeration, and/or social networks?", Urban Studies, 37, 3, pp. 513-32.

Gorman, M.E. (2002): "Types of Knowledge and Their Roles in Technology Transfer", Journal of Technology Transfer, 27, pp. 219-231.

Granovetter, M. (1985): "Economic action and social structure: the problem of embeddedness", American Journal of Sociology, Volume 91, Number 3, November 1985, pp. 481-510.

Granovetter, M. (1973): "The strength of weak ties", American Journal of Sociology, 81, pp. 1287-303.

Helliwell, J. F. (ed.) (2001): The contribution of human and social capital to sustained economic growth and well being. International Symposium Report, HRDC and OECD. 
Lawson, C. (1999): "Towards a competence theory of the region", Cambridge Journal of Economics, vol. 23, N. 2, March 1999, pp. 151-66.

Lundvall, B-A. (1992): National systems of innovation: toward a theory of innovation and interactive learning. London: Pinter.

Lundvall, B. (1994): "The learning economy: challenges to economic theory and policy". EAEP Conference.

Lundvall, B. (1996): "The social dimension of the learning economy", DRUID Working Paper, no 96-1.

Marshall, A. (1920): Principles of Economics. McMillan, London.

Morgan, K. (1997): "The learning region: institutions, innovation and regional renewal", Regional Studies, 31, 5, pp. 491-503.

Moreno, J.L. (1934): Who Sall Survive? in Foundations of Sociometry, Group Psychoterapy and Sociograma. Washington D.C.: Nervous and Mental Disease. Publishing.

Noguera, P. and M. Semitiel (2003): The Glue that Holds Economics and Sociology together. $6^{\text {Th }}$ Conference ESA, Spain.

Ouchi, W. G. (1980): "Markets, bureaucracies and clans", Administrative Science Quarterly, volume 25, issue 1 (March. 1980), pp. 129-41.

Peeters, L.; Tiri, M. and Berwert, A. (2001): "Identification of technoeconomic clusters using input-output data: applicaton to Flanders and Switzerland", in OECD (2001), Innovative cluster: drivers of national innovation systems, Paris, OECD; pp. 251-272.

Porter, M. E. (2003): "The economic performance of regions", Regional Studies, vol. 37, no67, pp. 549-78.

Putnam, R. (1993): "The prosperous community", The American Prospect Online, volume 4, Issue 13, March 21, 1993. Available in http://www.prospect.org/print/V4/13/putnam-r.html 
Putnam, R. D. y Goss, K. A. (eds.) (2002): Democracies in flux: the evolution of social capital in contemporary society. Oxfod: Oxford Press.

Rallet, A. y Torre, A. (1999): "Is geographical proximity necessary in the innovation networks in the era of global economy?", GeoJournal, 49, 4, pp. 373-80.

Scott, A. y Storper, M. (2003): "Regions, globalisation, development", Regional Studies, 37, 6\&7, pp. 579-93.

Storper, M. (1995): "Regional technology coalitions. An essential dimension of national technology policy", Research Policy, 24, pp. 895-911.

Storper, M. and Harrison, B. (1991): "Flexibility, hierarchy and regional development: the changing structure of industrial production systems and their forms of governance in the 1990s", Research Policy, 20, pp. 407-22.

Streit, M. E. (1969): "Spatial associations and economic linkages between industries", Journal of Regional Science, vol. 9, n. 2, pp. 177-88.

Wasserman, S. and Faust, K. (1999): Social network analysis, methods and applications. Cambridge: Cambridge University Press.

Wilkinson, F. (1983): "Productive systems", Cambridge Journal of Economics, Vol. 7, N. 3/4, September/December 1983, pp. 413-29.

Williamson, O. E. (1983): Markets and hierarchies. Analysis and antritrust implications. A study in the economics of internal organization. New York: The Free Press.

Woolcock, M. (2001): "The Place of Social Capital in Understanding Social and Economic Outcomes" in John F. Helliwell (ed.): The Contribution of Human and Social Capital to Sustained Economic Growth and Well-Being. International Symposium Report. HRDC and OECD, pp. 65-88. 